\title{
ETHIK
}

\section{Macht, Führung, Sinn}

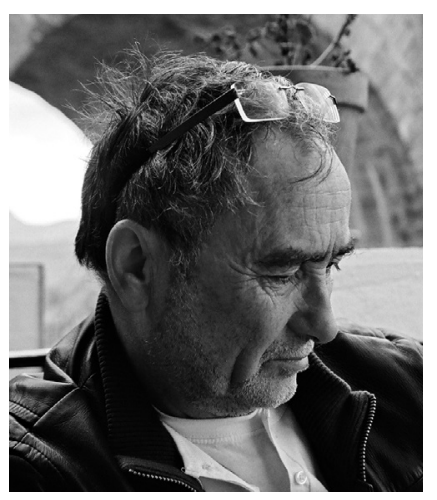

VON UTO MEIER

Prof. Dr. Uto Meier ist Hochschullehrer für Religionspädagogik an der Kath. Universität EichstättIngolstadt und wissenschaftliches Mitglied am Zentrum für ethische Bildung in den Streitkräften des Militärbischofsamtes. In Lehre und Forschung wie Weiterbildung beschäftigt er sich neben dem Kernfeld religiöser Identitätsbildung mit dem Spezifikum ethischer Verantwortung für authentische Führungskräfte.

www.ku.de/rpf/lehrpersonal/ uto-meier

\author{
Das Nachdenken über Ethos und Ethik gewinnt in \\ der Sozialwirtschaft zunehmend an Bedeutung. \\ Nachdem betriebswirtschaftliches Handeln \\ eingeführt und anerkannt ist, stellen sich \\ wieder vermehrt Fragen nach dem Sinn und der \\ Berechtigung sozialer Dienste und Einrichtungen.
}

Von Karl Kraus wird erzählt, dass er einen Studenten, den er seit Jahren nicht mehr gesehen hatte, fragte, was er denn nun studiere. Dieser habe stolz geantwortet: »Wirtschaftsethik«. Darauf Karl Kraus: »Da werden Sie sich entscheiden müssen!« (1)

Diese hintersinnige Anekdote macht vielleicht deutlich, dass die grundlegenden Spannungen im Bereich ökonomischen Erfolgs schon vor hundert Jahren bereits bewusst waren, nämlich die schwere Vereinbarkeit von Markt und Moral, von Ethik und Ebit, von Geist und Geld, oder heute von Umsatz oder Umwelt, wie die gängigen Dichotomien das Problem derzeit pointiert werden.

Diese Problematik ist keineswegs genuin für ökonomischen Erfolg indiziert, sie trifft auch - in abgewandelter Form den Bereich sozialwirtschaftlichen Erfolges, näherhin der Frage, welcher Führungsphilosophie und Führungsethik eine Institutionenleitung bedarf, um sich »erfolgreich« und gleichzeitig »verantwortet« zu verstehen.

\section{Der ethische Blick auf den Erfolg}

Mit Peter Ulrich (2) möchte ich den Erfolgsbegriff erweitern und in einer dreiwertigen Korrelation aufgehoben sehen (3): Als Formel hieße dies programmatisch verkürzt: Sinn + Gerechtigkeit + Effizienz $=$ integrer Erfolg.

Hier sind selbstredend komplexere Fragestellungen noch enthalten, etwa wie sich »Wertschöpfung « legitimiert (für wen? Mit welcher Berechtigung? Wer ist anspruchsberechtigt?), inwieweit Sinn in öffentlichen Diskursen hergestellt werden kann, und inwiefern nicht auch Effizienz, als Funktion einer Überlebensnotwendigkeit, gewisse Restriktionen mit sich bringt.

Gleichwohl dürfte klar sein, dass allein der Markt (unfraglich als EffizienzGarant für Herstellung und Verteilung) und seine Gesetze es eben nicht allein richten, wie Gammelfleisch und Mädchenhandel, wie Lehman-Pleite und die Widersprüche gänzlich unregulierter Finanzmärkte hinreichend belegen.

\section{Ethik ist kein Wert unter vielen}

Leider muss konstatiert werden, dass in der gegenwärtigen akademischen Debatte - nicht zuletzt in Unternehmen und Institutionen - nicht selten ein Kategorienfehler sich etabliert hat, der »Ethik« als Werte unter vielen begreift (4), und nicht als Maßstab, der alle Werte in eine verantwortbare Reihenfolge bringt.

Dahinter verbirgt sich ein nicht kleines philosophisches Problem, dass sich gleichsam »das Gute « durch »das Nützliche « ersetzen lasse, eine Position, die seit Georg Moore als »naturalistic fallacy« in der Literatur diskutiert wird.

Es ist schlichtweg ein widersprüchliches Verständnis von Ethik (jedenfalls in abendländischer Tradition), dass moralische Werte in manchen Unternehmensleitbildern als ein Wert neben anderen präferiert werden, sozusagen neben den Leistungswerten, neben den 
sozialen Werten und neben den Kommunikationswerten möge man auch noch ethische Werte beachten.

Es muss nämlich das Prädikat "gut« als »funktional gut " (gut für die Gesundheit, gut für den Umsatz, gut für das Unternehmenswachstum) gänzlich von einem »unbedingt ethisch gut « unterschieden werden, denn es kann durchaus umsatzgefährdende Kontexte geben, die ethisch geboten sind, wo der Marktaustritt verlangt werden darf, ja muss.

Dieser utilitaristischen Position eines - aus der Volkwirtschaftslehre kommenden (Jeremy Bentham und John Stuart Mill) - Moralansatzes hängt sein »Sitz im Leben« noch an: Es geht vermeintlich um Optimierung: »das größtmögliche Glück der größtmöglichen Zahl « heißt die allseits akzeptierte Ethik-Formel: »Diejenige Handlung bzw. Handlungsregel ist moralisch richtig, deren Folgen für das Wohlergehen aller Betroffenen optimal sind.«(5)

Hier müssen aber Einwände um des Menschen willen formuliert werden. Das Wohlergehen, der Nutzen aller, ist eine abstrakte Verantwortungsinstanz, deren wir gegenüber meinen rechenschaftspflichtig zu sein. Damit ist ein (lange tradiertes) personales Gegenüber durch ein Abstraktum ersetzt, dem wir nur ein allgemeines Optimieren schulden. Es wurde aber vielleicht evident (6), dass die Optimierungsstrategie das Opfern und die Verzweckung von Menschen nicht verhindern kann, ja eher fördert, ist doch am Ende die Bilanzierung eines Nutzens für viele oft höher.

Wahrscheinlich beruht die derzeit hohe Konjunktur des Verantwortungsbegriffes auch auf seiner klandestinen Konnotation, dass Moralität etwas Persönliches ist, denn Verantwortung ist ja eine Kategorie des »Face-to-face», ein Gegenbegriff zu den anonymen Mächten (und Märkten), die alle unsere Überzeugung und unser Gewissen gerne kaufen und gewinnen und anpassen wollen. Verantwortung ist eben ein Begriff des menschlichen Dazugehörens, eines KlarWerdens im Gespräch-Sein.

\section{Warum "ethikorientierte Führung" auch erfolgreicher ist}

Seit den Studien von Lawrence Kohlberg (7) ist deutlich geworden, dass eine »reife Moralität « aus bestimmten - man könnte sagen - vormoralischen Bewusstseinsstu- fen herauswächst, wenn die Kultur des Kontextes - das Unternehmen, die Institution - dies zulässt und fördert. Mit Peter Ulrich (8) will ich der Hypothese nachgehen, dass ein Unternehmen, eine Institution so gut ist, wie das moralische Bewusstsein ihrer Teilnehmer, insbesondere ihrer Führungsebene. Und dass diese »ethische Kultur« ebenso entwickelbar ist wie sie auch immer gefährdet bleibt, wenn die »oberste Heeresleitung « ihre Grundwerte nicht lebt und einfordert.

Für den Aufbau einer nachhaltigen Integritätskultur ist ein substantielles »Change-Management « (hier einmal anders verstanden), orientiert an moralischen Prinzipien, wesentlich, die tatsächlich (und nicht nur in den eingerahmten »Code of Conducts «, über die nicht selten lächelnd die wirklichen Deals abgewickelt werden) von überlebensrelevanter Bedeutung sind, wie viele Studien über die faktischen »Wert-Kulturen« von Firmen belegen. (9)

Kontinuierliche Verantwortungsbildung wäre so auch ein Schlüssel zum (langfristigen) Erfolg einer Unternehmung wie dem Aufbau einer humanen Zivilgesellschaft.

\section{Nicht jeder ist für alles verantwortlich - aber alle sind für das ihre verantwortlich}

Gemeinhin (10) wird der Verantwortungsbegriff in der neueren Debatte in einer vierstelligen Relation begriffen:

(1) Ein Verantwortungssubjekt (Person, Firma, Institution) ist für ein

(2) Verantwortungsobjekt (Handlungen, Aussagen) gegenüber einer

(3) Verantwortungsinstanz (Gericht, Gewissen, öffentliche Meinung) vor einem

(4) normativen Hintergrund (Regelwerk als Kann- oder Soll- oder Muss-Regel, Qualität einer sittlichen Beziehung, deontologischer versus teleologischer Normenbegründung) verantwortlich.

Damit ist allerdings noch nicht das wesentliche Moment des zugrunde liegenden Handlungsverständnisses geklärt. Denn auch Mafia-Mitglieder »verantworten« sich, indem sie persönlich für die einzutreibenden Schutzgelder haften und vor dem »Ehrenrat« ihrer »Ehrenwerten Gesellschaft « sich rechtfertigen müssen. Ohne scharfe Klärung der ethischen Legitimität des normativen Hintergrundes ist also noch nicht viel für eine substantielle ethische Betrachtung gewonnen, es bliebe bei einem problematischen Relativismus, wenn der normative Hintergrund nicht aus seiner funktionalen Begründung (will sagen: gut als nur »gut-für-etwas «) befreit wird.

$\mathrm{Zu}$ einem sittlichen Handlungsakt gehört immer schon das Wissen um die Handlungsfolgen (und ihres Rechtsoder Unrechtscharakters) sowie der bewusste und freie Vollzug, dessen (Langzeit-) Folgen also noch dem Subjekt zuzuschreiben sind.

Und dennoch gilt es zu bedenken, dass nicht jeder für alles verantwortlich sein kann und darf, allein wegen der unterschiedlichen Kompetenzen (und damit Einblicksreichweiten), die die Vorhersehbarkeit beeinflussen. Und natürlich vor der zugewiesenen Macht, die unterschiedlichen Entscheidungshöhen zur Voraussetzung haben.

Deswegen muss der Verantwortungsbegriff im Blick auf seine Verantwortungshöhe bedacht werden. Mit einem systemischen Blick unterscheidet man seit Luhmann eine

a) Verantwortungsethische Mikroebene unmittelbarer personaler Interaktion (individuelle Verantwortlichkeit in der konkreten Sacharbeit, als methodischer Sorgfalt, Pflichterfüllung gegenüber den Rollenerwartungen, z. B. der unmittelbaren Gefahrenvermeidung wie auch Auftragserfüllung) und damit unmittelbarer Verantwortung vor den Zielen der beruflichen wie außerberuflichen Verpflichtungen. Die Reinemachefrau in einem Altersheim ist nicht für ein problematisches schlechtes Betreuungskonzept der Einrichtung zuständig, auch wenn sie die technisch-hygienische Voraussetzungen für den Betrieb der schlechten Einrichtung mitgestaltet.

b) Verantwortungsethische Mesoebene als die Verantwortung der Organisation(sziele), in der das Individuum steht. Auch ein Arzt kann sich in Forschung und im Vollzug - um die Gesundheit eines Folterkandidaten kümmern - und damit seinen Job "gut" machen -, aber er sollte eben gar nicht in der Struktur eines die Personenwürde verachtenden Regimes arbeiten oder sich wissenschaftlich vorgängig dazu engagieren. 
c) Verantwortungsethische Makroebene, die den grundsätzlichen Fragen nachgeht, inwieweit beispielsweise Wissenschaft sich welchen Zielen unterordnen darf (Wissenschaft als von Zweck und Verwendung unabhängig verstanden, gar blind fortschrittsfixiert (ohne die Qualität von Fortschritt - und Fortschritt für wen? - zu reflektieren); diese Makroebene ist daher als Reflexion der (finanziellen wie philosophischen) Rahmenbedingungen $\mathrm{zu}$ verstehen, unter denen beispielsweise Sozialwissenschaft sich bestimmten Themenfeldern widmet (Kernphysik oder Armutsbekämpfung? Wirtschaftswissenschaft als Lehre von Gewinnmaximierung oder Gemeinwohlmaximierung etc.). Letztlich geht es hier um eine Allokationsethik von Ressourcen, denn in der heutigen Forschungslandschaft wie der Sozialwirtschaft kommt nur voran, wer Ressourcen zugewiesen bekommt. Und diese Ressourcenzuweisungen sind ethisch begründungspflichtig für Entscheider.

Wenn diese Differenzierung unterschiedlicher Verantwortungsebenen richtig ist, gelten eben auch unterschiedliche Verantwortungsgrade: Der Doktorand sollte einen Versuch nach Weisung seines Doktorvaters ausführen - er kann nämlich meist nicht wissen, dass eventuell seine Ergebnisse á la longue missbraucht etwa im Leitziel der Menschenwürde gefasst sind. (11) Damit ist deutlich geworden, dass Verantwortlichkeit jeweilig ist, sie kann nicht so einfach vor einem einzigen Organisationsziel legitimiert werden - beispielsweise dem der Profitabilität oder nur dem ideal Wünschenswerten sondern sie muss sich über ihren Rechtfertigungsgrund Gedanken machen.

\section{Ethikorientierung in der Sozialwirtschaft: Was ist die elementar-essentielle Aufgabe einer Einrichtung?}

Ich möchte hier für einen moralphilosophischen Ansatz werben, der an die fast vergessene Überzeugung erinnern will, dass »das Gute das ist, was der Wirklichkeit gerecht wird « (12) oder in alter Terminologie: Das Handeln folgt dem Sein (agere sequitur esse).

Ethik wäre so zuerst »Wirklichkeitsaufmerksamkeit (Spaemann) und keine wie auch immer geartete Zweckoptimierung und utilitaristische Zielrealisierung, etwa in Gestalt der Forderung, dass alle sozialen Institutionen letztendlich »Profit-Center" sein müssten. Mit Robert Spaemann kann man sagen, dass Ethik "nichts anderes, als die richtige, die wirklichkeitsgemäße Ordnung der Sachgesichtspunkte« (13) ist. Es geht also um das elementare Sollen, das die wesensgemäße (essentielle) Forderung einer Sache legitimiert, daher Elementar-Essentialismus genannt.

\section{"Die These sei gewagt: Ein Unternehmen}

\section{ist so gut wie das moralische}

\section{Bewusstsein seiner Führungsebene»}

werden; der Ordinarius muss sich schon intensiver fragen, welche Forschungsziele er anstreben kann, wem und wie seine Forschung dient und dienlich ist; und die Wissenschaftspolitik(er) wie die »scientific community « müssen sich fragen lassen, vor welchem Menschenbild und vor welchen Wertpräferenzen sie Wissenschaft betreiben, wenn sie Milliarden in bestimmte Richtungen lenken oder Milliarden anderen Zielen vorenthalten.

Alle Ebenen müssen sich aber absoluten Mindeststandards rechtfertigen, die nie unterschritten werden dürfen, wie sie
Ein Elementar-Essentialismus begründet eine Norm nicht primär mit Nutzenoptimierung, sondern mit der Verpflichtung aus dem Wesen einer Sache oder Beziehung: Es liegt eben beispielsweise im Wesen des Richters, dass er unabhängig Recht spricht; niemand käme auf den Gedanken zu fragen, ob das Gericht zuerst »rentabel « sein muss; und es liegt im Wesen der Kommunikation, dass sie wahrheitsapproximativ angelegt ist; und kein verantwortlicher Mensch käme auf den Gedanken, zuerst zu fragen, ob eine Aussage primär nütz- lich ist; und das Wesen des Heilungsauftrages oder der Leidensminderung für den Arzt ist nicht durch irgend ein äußeres - grundsätzlich auch Sinnvolles - , wie beispielsweise Kostenersparnis bestimmt, sondern eben zuvörderst durch den Heilungsauftrag gegenüber einem Menschen begründet.

Es lassen sich - nicht zuletzt im Bereich der Sozialwirtschaft - nicht wenige Segmente identifizieren, die sich eben nicht aus »Markttauglichkeit « legitimieren (dürfen), sondern aus »Sinn-Tauglichkeit«. Und dafür muss die öffentliche Hand Verantwortung tragen.

Selbstredend schließt dies Wirtschaftlichkeitsdenken ein, dient ja gerade sorgfältig zielgebundene Mittelverwendung dem Auftrag einer sozialen Einrichtung. Und es ist durchaus kein Sakrileg, wenn soziale Einrichtungen ihr Knowhow auch einem interessierten Markt zur Verfügung stellen. Aber ihre Legitimierung sollte nicht primär aus ihrer Vermarktbarkeit abgeleitet werden, wenn sie als unaufgebbarer Auftrag für eine menschengemäße Sozietät (von der Politik!) definiert wurde.

Und das bedeutet dann für eine verantwortbare Finanzierung, dass die ökonomische Basis einer Einrichtung nicht primär Marktgesetzen ausgeliefert sein darf. Daher ist - gerade im Blick auf das starke Wirtschaftlichkeitsgebot für die Sozialwirtschaft - sorgfältigst zu klären, ob eine Einrichtung als Service-Angebot zu verstehen ist, die im Markt bestehen kann - oder auch nicht -, oder ob das Wesen und die Aufgabe einer Einrichtung aus sich heraus geboten, und nicht primär aus seiner Markttauglichkeit zu legitimieren ist.

\section{Ethikstandards in der Personalführung}

Aus dem Gesagten ergibt sich, dass Führungskonzepte in der Sozialwirtschaft weniger den Schlagworten einer eindimensionalen Erfolgsphilosophie nachlaufen sollten, deren Sterne schon länger nicht mehr so recht leuchten, wenn von Mitarbeitern als Objekte, als Humankapital oder Human resource gesprochen wird. In dieser einseitigen Perspektive dominiert als Beziehungsmuster ein MittelZweck-Denken. Hier sind eher individualistische und primär ökonomi(sti)sche Werte vorherrschend, die Person wird oft nur als Kostenfaktor identifiziert, letzt- 
lich ist das Endziel Gewinnmaximierung. Der Prophet dieser neuen Religion ist bekanntlich Milton Friedman.

Anders hingegen ist der Ansatz von »ethikorientierter Führung « fundiert, wie er etwa von unter anderem von Dieter Frey (14) propagiert wird: Mitarbeiter werden als Subjekte gesehen, deren legitime Bedürfnisse wahrgenommen werden (Autonomie und Entwicklungschancen werden respektiert). Beziehung wird hier unter Selbstzwecklickeit (nach Kant) verstanden, die in transparenter wie kongruenter und zielorientierter Kommunikation gelebt wird; die legitimen Aufgaben und Visionen dient, aber keineswegs von diesen ausschließlich bestimmt ist. Grundrechte und Grundpflichten dominieren und Personen werden in ihrem Entwicklungspotential gesehen. Als Endziel der Mitarbeiterführung steht »Sinnstiftung «, die unter gelebter klarer Fairness (operationalisiert auch in einer erfolgs- wie bedürfnisbezogenen Entlohnung) realisiert wird.

Diese Form sach- wie mitarbeiterbezogener Leadership zeitigt eindeutig und zwar evidenzbasiert (15) - bessere Betriebsergebnisse. Sie erweist sich förderlich für den (langfristigen) Unternehmenserfolg wie für die Berufszufriedenheit aller Beteiligten. Die Maxime einer »ethischen Führung " lautet so auch: Wertschöpfung durch Wertschätzung!

Aus den sozialpsychologischen Erkenntnisgewinnen der Arbeitspsychologie wie auch aus dem langen Gedächtnis guter Führungstraditionen etwa der Klöster (16) lässt sich resümieren, wie ein Erfolg entstehen kann, der nachhaltiger und umfassender als kurzfristig nur ökonomischer Gewinn ist, denn dieser ruht auf Zielen wie Reputation, Akzeptanz und Fairness auf wie:

- Schätze sehen lernen, nicht Fehler suchen!

- Win-win-Lösungen anstreben!

- Sachebene von Personenebene trennen!

- Aufrichtig (kongruent) sich mitteilen!

- Feedback: sachorientiert geben, ohne Abwertung!

- Einbinden, nicht raus stellen!

- Verantwortungen eindeutig klären!

- Vergeben lernen (»Everyboby needs a second chance!)

- Mit dem Theologen und Unternehmensberater Ulrich Hemel könnte man als Guidelines guter, also ethisch basierter Institutionenleitung folgende Standards formulieren (18):
- Professionalität als Wertschöpfung

- Tue das gut, was du tust! Unternehmungen und Institutionen müssen legitime Bedürfnisse erfüllen und auftragskongruent sein! Gut gemeint ist noch nicht gut!

- Vertrauen und Wertschätzung: Es gilt ein grundsätzliches »Du bist o.k.«: Erfülle das in Dich gesetzte Vertrauen aus den legitimen (Rollen-) Erwartungen an Dich und gewähre anderen Vertrauen und Anerkennung!

- Transparente Kommunikation: Nur Kongruenz bindet nachhaltig! Wahrhaftigkeit ist Voraussetzung aller gelingenden Interaktion, aber mit unterschiedlicher Zugehörigkeit in Diskursorten! Diskretion und Offenheit müssen angemessen ausbalanciert sein!

- Zielklarheit und Strategie: Transparenter Kurs ist gefordert! Alle Leitenden haben die Pflicht, ihren »Kurs" $\mathrm{zu}$ artikulieren und alle Betroffenen das Recht, Kursvorgaben und Ziele zu bejahen oder zu befragen.

- Persönliche Verantwortung: Zuschreibung. Aus Achtung vor Mitarbeitern und im Dienst der Sache muss persönliche Verantwortung gelten, mit geklärter Verantwortungsreichweite und deutlicher Verantwortungs(ab) stufung!

- Personenwürde: Personalität achten ist letzter Maßstab! Die personale Würde ist immer zu achten: Kein Zweck heiligt die Verletzung des Personenkerns!
Anschließend soll in einem resümierenden Überblick eine ethische Kriteriologie entworfen werden, die als Maßstab für das gelten kann, was nicht mehr einem »Gut für etwas « unterworfen werden, sondern als Richtlinie für ein »Gut an sich « herangezogen werden kann. Der Meier-Pagano-Filter (18) will eine primäre ethische Entscheidungshilfe darstellen, die Führungskräften aus der großen abendländischen Tradition eine prinzipienbasierte Unterscheidungshilfe eröffnet (vgl. Abb.).

Entscheidungsgrundlegungen mit diesen Prinzipien werden schwerlich noch Handlungen legitimieren, die sich unter den Maßstab moralisch begründeter, also ethischer Verantwortung stellen wollen.

\section{Implementierung einer "ethischen Führungskultur"}

Neben den Faktoren einer notwendigen Proklamation von entsprechenden Führungsrichtlinien, neben MultiplikatorenGewinnung und der unbedingten klaren und transparenten Führungsauthentizität der Leitung, braucht es selbstredend eine Diagnose vorhandener Integritätslücken, die die Soll-Bruchstellen zwischen den drei großen Ebenen einer Unternehmenskultur der "Artefacts « (sichtbare Strukturen und Zeichen), der "Values « (Öffentlich propagierter Werte) und den »Basic assumptions « (normative Hintergrundannahmen), betreffen, die jede Unternehmenskultur ausmacht. (19)
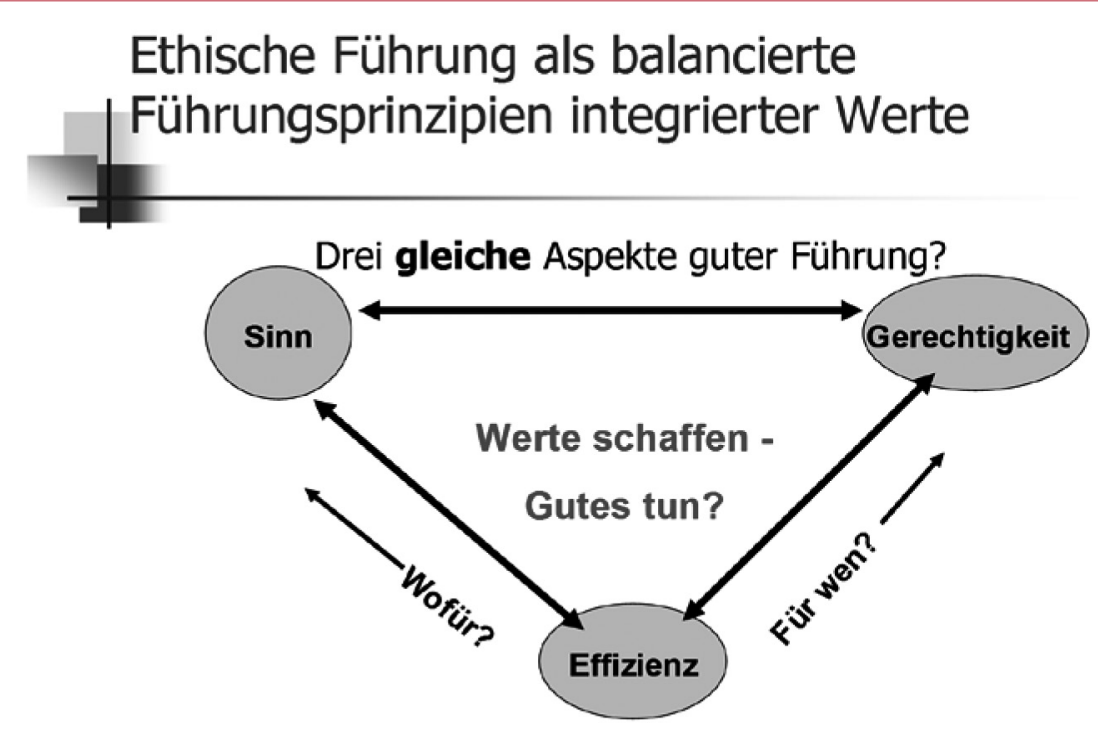

Der Meier-Pagano-Filter soll eine ethische Entscheidungshilfe sein, mit dem Führungskräften auf dem Hintergrund abendländischer Tradition eine prinzipienbasierte Unterscheidungshilfe eröffnet wird. 
Diese normativen Hintergrundannahmen (»Woran glauben wir wirklich?«) und dem, was gelebte Institutionenkultur ist (»Wie wir wirklich handeln und entscheiden!«), müssen erkannt und bearbeitet werden. Desgleichen sollte die Differenz zwischen dem vereinbarten (und erarbeiteten!) Code of Conduct und dem tatsächlichen beruflichen Handeln analysiert und gegebenenfalls problematisiert werden. Schlussendlich sollte dem »Moral point of view « ständig ein Forum gegeben werden, besser eine Stelle (den Ethic-Officer), die die Einhaltung der »Verfassung « einer Einrichtung zu seiner ureigenen (Teil-)Aufgabe machen kann.

Dieser Prozess sollte auf individuellen Ebenen installiert werden (Moralische Urteilsbildung wie Zivilcourage-Anreize), dieser Prozess braucht aber auch organisationale Rahmenbedingungen (eine offene Integritätskultur, eine klare Anlaufstelle für Dilemma-Situationen) und - last baut not least - eine Führung, die wirklich eine Kultur der Integrität und der Verantwortung als Basis aller institutionalen Zielverwirklichung will.

Nur dann macht eine ethische Weiterbildung, die Arbeit an einem gelebten wie lebbaren Code of conduct und viele weitere Engagements für eine Kultur der Integrität Sinn. Führung macht eben nur dann Sinn, wenn Macht über Führung (nach-) sinnt.

Bei dem Artikel handelt es sich um die überarbeitete Kurzfassung des gleichnamigen Vortrages auf der ConSozial 2012.

\section{Anmerkungen}

(1) Zitiert nach: Uto Meier/Bernhard Sill (Hg), Führung.Macht.Sinn. Ethos und Ethik für Entscheider in Wirtschaft, Gesellschaft und Kirche. Regensburg (Pustet Verlag) 2010, S. 5. In diesem Sammelband sind wesentliche Positionen der hier nur kurz skizzierbaren Führungsethik breit diskutiert.

(2) Peter Ulrich, Der entzauberte Markt. Eine wirtschaftethische Orientierung. Freiburg i. Br. 2002. Auch: Thomas Maak/Peter Ulrich, Integre Unternehmensführung. Ethisches Orientierungswissen für die Wirtschaftspraxis. Stuttgart 2007.
(3) Vgl. dazu Peter Ulrich 2002, S. 30, wo er mit dem Begriff »wirtschaftliche Vernunft « bereits wesentliche Kategorien der Ethik integriert: Sinn und Gerechtigkeit und Effizienz als drei Aspekte eines umfassenden Erfolgsverständnisses, das er zu einem integrierten Verständnis von "Wertschöpfung « erweitert.

(4) Tendenziell etwa bei Wieland, Josef (2006): Gesellschaftliche Verantwortung der Unternehmen. In: Unternehmensethik im Spannungsfeld der Kulturen und Religionen. Stuttgart, S. 2-10 zu beobachten, vgl. das Schaubild S. 8 .

(5) Höffe, Otfried (2003): Einführung in die utilitaristische Ethik. Tübingen, 3. Auflage, S. 11.

(6) Spaemann 2001: Über die Unmöglichkeit einer universalteleologischen Ethik, S. 193-212.

(7) Vgl. zur Kohleberg-Theorie: Fritz Oser/Wolfgang Althof, Moralische Selbstbestimmung. 4. Auflage 2001.

(8) Thomas Maak/Peter Ulrich, Integre Unternehmensführung. Ethisches Orientierungswissen für die Wirtschaftspraxis. Stuttgart 2007, hier Kapitel/Modul 14, Ethische Kompetenzbildung, S. 471-493.

(9) Vgl. dazu Thomas Maak Peter Ulrich, Integre Unternehmensführung, a. a. O., hier Kapitel/Modul 9: Responsible Change \& Moralisches Lernen, hier besonders Seiten 327-329 über »Moralische Reifegrade von Unternehmen «.

(10) Zusammenfassend: Assländer, Michael; Grundlagen der Wirtschaftsund Unternehmensethik. Marburg 2011.

(11) Vgl. dazu: Meier, Uto: Über Grenzen und Leitplanken: ethische Verantwortung heute - jenseits zweckrationaler Vernunft; elementarmoralische Einwürfe gegen eine Ethik der Güterabwägung ; ein Plädoyer für eine Rehabilitation des »von Natur aus Rechten«. In: Kontakt : Informationen zum Religionsunterricht im Bistum Augsburg. (2012) Heft 4. S. 7-20.

(12) So Spaemann 1982, S. 91.

(13) Spaemann 1982, S. 89.

(14) Vgl. dazu zusammenfassend: Dieter Frey u. a., Unternehmenserfolg durch ethikorientierter Unternehmens- und Mitarbeiterführung. In:
Uto Meier/Bernhard Sill (Hg), Führung.Macht.Sinn. Ethos und Ethik für Entscheider in Wirtschaft, Gesellschaft und Kirche. Regensburg 2010, hier: 637656.

(15) Dieter Frey, ebd. Belegt mit sozialwissenschaftlichen Evaluierungen, vgl. Literatur dazu: 654-656.

(16) Vgl. dazu: Stefan Kiechle SJ, Mitarbeiter führen. Anregungen aus den Satzungen des Jesuitenordens. In Meier/Sill 2010, S. 501-512. Auch: Anselm Bilgri, Wege zum Sinn-Erfolg. Die Benediktsregel als Richtschnur einer verantwortungsbewussten Unternehmensführung. In: Meier/Sill 2010, S. 525-536. Notger Wolf OSB, Alter Wein in neue Schläuche. Moderne Führungsverantwortung auf der Basis der Regel Benedikts von Nursia. In: Meier/Sill 2010, S. 379-393.

(17) Ulrich Hemel, Wirtschaftlichen und ethischen Mehrwert schaffen. Führen mit werten und Zielen. In: Uto Meier/Bernhard Sill (Hg.), Zwischen Gewissen und Gewinn. Werteorientierte Personalführung und Organisationsentwicklung. Regensburg 2005, hier: S. 144-156.

(18) Erstmalig in: Uto Meier: Über Grenzen und Leitplanken : ethische Verantwortung heute - jenseits zweckrationaler Vernunft ; elementarmoralische Einwürfe gegen eine Ethik der Güterabwägung ; ein Plädoyer für eine Rehabilitation des „von Natur aus Rechten“. In: Kontakt : Informationen zum Religionsunterricht im Bistum Augsburg. (2012) Heft 4. S. 7-20, hier S. 20.

(19) Edgar H. Schein, Organizational Culture and Leadership, Hoboken N.Y. 1987, zitiert nach der deutschen Ausgabe, Bergisch Gladbach 2003, hier S. 31. 